\section{Vitamin D in COVID-19 Pneu- monia: High Yield with Low Risk}

\section{Sir,}

The novel coronavirus disease 2019 (COVID-19) is a global public health problem with particularly poor outcomes in the elderly. The disease pathogenesis and presentation is still evolving. Vitamin D is a fat-soluble, secosterol that exists in two forms: vitamin D2 and D3. It is obtained mostly from food with a small portion synthesised by sunlight. The association of low vitamin D levels with increased susceptibility to upper respiratory tractinfections (URTIs) is known in the literature. ${ }^{1}$ A meta-analysis did show benefit of taking vitamin $D$ supplementation among patients with low 25 hydroxy-vitamin D levels presenting with URTIs. ${ }^{3,4}$ There are studies suggesting utility of vitamin D both in prevention, and in decreasing severity and mortality among patients with COVID-19 pneumonia. The mechanism is linked to development of antimicrobial peptides and activation of dendritic cells, macrophages, Tand B lymphocytes through utilisation of vitamin $D$ receptors (VDR) that improve immunity and decrease inflammatory response against COVID-19. ${ }^{4,5}$ The virus interacts with ACE2 receptors on type II pneumocytes and leads to cell death. Vitamin $D$ also has immunomodulatory properties that downregulate activity of pro-inflammatory cytokines and further suppress ACE2 receptor activity. ${ }^{4,5}$ This protective effect of vitamin $\mathrm{D}$ can be achieved by a single oral vitamin D3 dose (2000 IU).

TheCOVID-19 disease severity may belinked to location of countries, which in turn, determines the extent of sunlight exposure. In this regard, it is reported that dermal production of vitamin $D$ from solar UVB between October to March is low above 35 degrees latitude. Countries included in these regions are predominantly inhabited by elderly, who are predisposed to higher probability of cardio-pulmonary and metabolic comorbidities. This might be a reason for such high incidence and mortality from COVID-19 in Western Europe and America. The cross-sectional analysis of 20 European countries does prove the notion that majority of people who suffered death were elderly and likely vitamin D deficient, based on data analysis from those regions. The problem is exacerbated during prolonged lockdown, where people are not getting enough sunlight exposure and; hence, stressed on already deficient vitamin D levels.

Vitamin $D$ can be used as supplementary therapy in patients to boost up the innate immunity and to combat mild to moderate COVID-19 pneumonia. Micronutrient supplementation including vitamin $\mathrm{D}$ and $\mathrm{C}$ should be started early in the disease process. Though vitamin D utility in COVID-19-related pneumonia has not been evaluated, trials are underway to further test its efficacy in COVID-19-associated pneumonia; and the results are expected in the coming months. The current treatments in use are very expensive and need validation through superiority designs. Early administration of vitamin D may prove beneficial in reducing the severity of symptoms and possibly hospitalisation rates in low resource settings like Pakistan.

\section{CONFLICT OF INTEREST:}

Authors declared no conflict of interest.

\section{AUTHORS' CONTRIBUTION:}

SW, ES: Contributed to conceptualization and write-up of the study.

\section{REFERENCES}

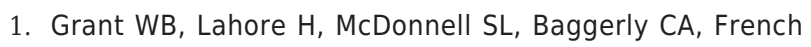
$C B$, Aliano JL, et al. Vitamin D supplementation could prevent and treat influenza, coronavirus, and pneumonia infections. Nutrients 2020; 12(4):988. doi: 10.3390/nu 12040988.

2. Cortegiani A, Ingoglia G, Ippolito M, Giarratano A, Einav S. A systematic review on the efficacy and safety of chloroquine for the treatment of COVID-19. J Critical Care 2020; 57: 279-83. doi: 10.1016/j.jcrc.2020.03.005.

3. Grant WB, Lahore $H$, McDonnell SL, Baggerly CA, French $C B$, Aliano JL, et al. Evidence that vitamin $D$ supplementation could reduce risk of influenza and COVID-19 infections and deaths. Nutrients 2020; 12(4): 988. doi: 10.3390/nu12040988.

4. Daneshkhah A, Agrawal V, Eshein A, Subramanian H, Roy HK, Backman V. The possible role of vitamin $D$ in suppressing cytokine storm and associated mortality in COVID-19 patients. MedRxiv 2020; doi.org/10.1101/ 2020.04.08.20058578.

5. Daneshkhah A, Eshein A, Subramanian H, Roy HK, Backman $V$. The role of vitamin $D$ in suppressing cytokine storm in COVID-19. patients and associated mortality. MedRxiv 2020; doi.org/10.1101/2020.04.08.20058578.

Shahan Waheed and Emaduddin Siddiqui

Department of Emergency, The Aga Khan University Hospital, Karachi, Pakistan

Correspondence to: Dr. Shahan Waheed, Department of Emergency, The Aga Khan University Hospital, Karachi, Pakistan

E-mail: docshahan83@hotmail.com

Received: August 23, 2020; Revised: October 23, 2020;

Accepted: October 23, 2020

DOI: https://doi.org/10.29271/jcpsp.2020.JCPSPCR.CR180 